\title{
Functional interplay between p63 and p53 controls RUNX1 function in the transition from proliferation to differentiation in human keratinocytes
}

\author{
I Masse ${ }^{*, 1}$, L Barbollat-Boutrand ${ }^{1}$, M Molina ${ }^{1}$, O Berthier-Vergnes ${ }^{1}, \mathrm{~N}$ Joly-Tonetti ${ }^{1}$, MT Martin ${ }^{2}$, C Caron de Fromentel ${ }^{3}$, \\ J Kanitakis ${ }^{4}$ and J Lamartine ${ }^{*, 1}$
}

The interfollicular epidermis is continuously renewed, thanks to a regulated balance between proliferation and differentiation. The $\Delta \mathrm{Np} 63$ transcription factor has a key role in the control of this process. It has been shown that $\Delta$ Np63 directly regulates Runt-related transcription factor 1 (RUNX1) transcription factor expression in mouse keratinocytes. The present study showed for the first time that RUNX1 is expressed in normal human interfollicular epidermis and that its expression is tightly regulated during the transition from proliferation to differentiation. It demonstrated that $\Delta \mathrm{Np} 63$ directly binds two different RUNX1 regulatory DNA sequences and modulates RUNX1 expression differentially in proliferative or differentiated human keratinocytes. It also showed that the regulation of RUNX1 expression by $\Delta$ Np63 is dependent on p53 and that this coregulation relies on differential binding and activation of RUNX1 regulatory sequences by $\Delta$ Np63 and p53. We also found that RUNX1 inhibits keratinocyte proliferation and activates directly the expression of KRT1, a critical actor in early keratinocyte differentiation. Finally, we described that RUNX1 expression, similar to $\triangle N p 63$ and p53, was strongly expressed and downregulated in basal cell carcinomas and squamous cell carcinomas respectively. Taken together, these data shed light on the importance of tight control of the functional interplay between $\triangle \mathrm{Np} 63$ and p53 in regulating RUNX1 transcription factor expression for proper regulation of interfollicular epidermal homeostasis.

Cell Death and Disease (2012) 3, e318; doi:10.1038/cddis.2012.62; published online 7 June 2012

Subject Category: Cancer

The epidermal homeostasis relies on proper control of proliferation and differentiation programs within epidermal cells. This differentiation process requires a precise coordination of the molecular events maintaining functional epidermis. p63 belongs to the p53/p63/p73 family of transcription factors (TFs) that share a conserved DNA-binding domain and similar DNA target sites in regulatory sequences of eukaryotic genes. p63 is essential for the development and maintenance of pluristratified epithelia, including epidermis. ${ }^{1}$ Mice lacking p63 display profound defects in epidermis formation and died shortly after birth due to dehydration. ${ }^{2,3}$ p63 is also critical for postnatal proliferation in basal keratinocytes and epidermal differentiation. ${ }^{4}$ Several p63 proteins have been described, resulting from two distinct promoters, TAp63 and $\triangle \mathrm{Np} 63$, and from alternative mRNA splicing at the $3^{\prime}$ end of the gene. ${ }^{5}$ The major isoform present in adult human epidermis is $\Delta \mathrm{Np} 63 \alpha$, expressed at high levels in the basal layer and at lower levels in suprabasal keratinocytes. ${ }^{6}$ p63 is overexpressed in many epithelial tumors, namely basal cell carcinoma (BCCs) and squamous cell carcinomas (SCCs) ${ }^{7}$ and is mutated in $8 \%$ of head and neck SCCs. ${ }^{8}$
Runt-related transcription factor 1 (RUNX1) has been identified among the direct p63 transcriptional targets in epidermis. ${ }^{9-11}$ RUNX1 belongs to the RUNX TFs family, which have an essential role in the balance between cell proliferation and differentiation in animal tissue development. RUNX family genes share a region, the Runt domain, which, with its partner subunit core-binding factor beta $(\operatorname{CBF} \beta)$, is responsible for specific DNA binding and heterodimerization and activates or represses transcription through the recruitment of various coactivators and corepressors depending on the cellular context. ${ }^{12}$ The RUNX genes also have a complex role in cancer, acting as either tumor suppressors or dominant oncogenes. ${ }^{13}$ RUNX1 is a master regulator in the development, growth and differentiation of hematopoietic, ${ }^{14}$ muscular ${ }^{15}$ and nerve cells. ${ }^{16}$ In mouse skin, RUNX1 expression was found in hair follicles, and its specific disruption in mouse epidermal cells impaired hair follicle homeostasis and long-term differentiation. ${ }^{17-19}$ In human skin, RUNX1 is also highly expressed in hair follicles and involved in KAP5 regulation, a keratin-associated protein expressed in the cuticle layer of anagen hair follicles. ${ }^{20}$

\footnotetext{
${ }^{1}$ Centre de Génétique et de Physiologie Moléculaires et Cellulaires, CNRS UMR5534-Université Lyon I, 43 Bd du 11 Novembre 1918, F-69622 Villeurbanne, France; ${ }^{2}$ Laboratoire de Génomique et Radiobiologie de la Kératinopoïèse, CEA, iRCM, Evry F-91000, France; ${ }^{3}$ Centre de Recherche en Cancérologie-Lyon Est, INSERM, U1052, CNRS, UMR5286, F-69008 Lyon, France and ${ }^{4}$ Department of Dermatology, Edouard Herriot Hospital Group (Pavillon R), F-69008 Lyon, France

${ }^{*}$ Corresponding authors: I Masse and J Lamartine, Centre de Génétique et de Physiologie Moléculaires et Cellulaires, CNRS UMR5534-Université Lyon I, 43 Bd du 11 Novembre 1918, F-69622 Villeurbanne, France. Tel: +33 4724329 50; Fax: +33 4724326 85; E-mail: ingrid.masse@univ-lyon1.fr (IM) or jerome.lamartine@univ-lyon1.fr (JL)

Keywords: RUNX1; p63; p53; keratinocytes; carcinoma

Abbreviations: RUNX1, Runt-related transcription factor 1; TF, transcription factor; BCC, basal cell carcinoma; SCC, squamous cell carcinoma; CBF $\beta$, core-binding factor beta; KAP5, keratin-associated protein 5; ChIP, chromatin immunoprecipitation; QPCR, quantitative PCR; siRNA, small interfering RNA; PCNA, proliferation cell nuclear antigen; KLF4, Krueppel-like factor 4

Received 14.12.11; revised 24.4.12; accepted 02.5.12; Edited by E Candi
} 
To date, RUNX1 function has never been explored in human interfollicular epidermis. We therefore investigated RUNX1 expression pattern in human normal skin, revealing expression in the interfollicular epidermis. Our data shed light on the complex coregulation of RUNX1 by $\Delta$ Np63 and p53, leading to a role for RUNX1 in the switch from proliferation to differentiation by repressing the cell cycle and activating a marker of early keratinocytes differentiation. It also showed that RUNX1 expression is dysregulated in human cutaneous BCCs and SCCs.

\section{Results}

RUNX1 is expressed in normal human interfollicular epidermis and tightly regulated in primary keratinocytes. RUNX1 gene expression is restricted to hair follicles in mouse $\operatorname{skin}^{18}$ and is strongly expressed in human hair follicles. ${ }^{20}$ We therefore investigated whether RUNX1 was also expressed in human interfollicular epidermis, using a
RUNX1-specific antibody on sections of normal human skin (Figure 1). As previously described, a strong RUNX1 expression was observed in cell nuclei from both the outer root sheath and the inner root sheath of human anagen hair follicles (Figures 1a and b). RUNX1 was also detected at lower but consistent levels in interfollicular epidermis: most of the basal keratinocytes exhibited specific nuclear labeling (Figures 1c and d) that decreased in suprabasal layers, when compared with negative control (Figure 1e). This staining pattern was observed in foreskin, facial, breast and abdominal skin.

To investigate RUNX1 expression more precisely in normal human interfollicular keratinocytes, we next used a model of $\mathrm{Ca}^{2+}$-induced differentiation of human primary keratinocytes (HPK) in culture, monitored by induction of keratin1 (Figure 2a), keratin10 (Figure 2b), involucrin (Figure 2c) and loricrin (Figure 2d), widely used markers of epidermal differentiation. At both mRNA (Figure 2e) and protein (Figure 2f) levels, RUNX1 was tightly regulated along the
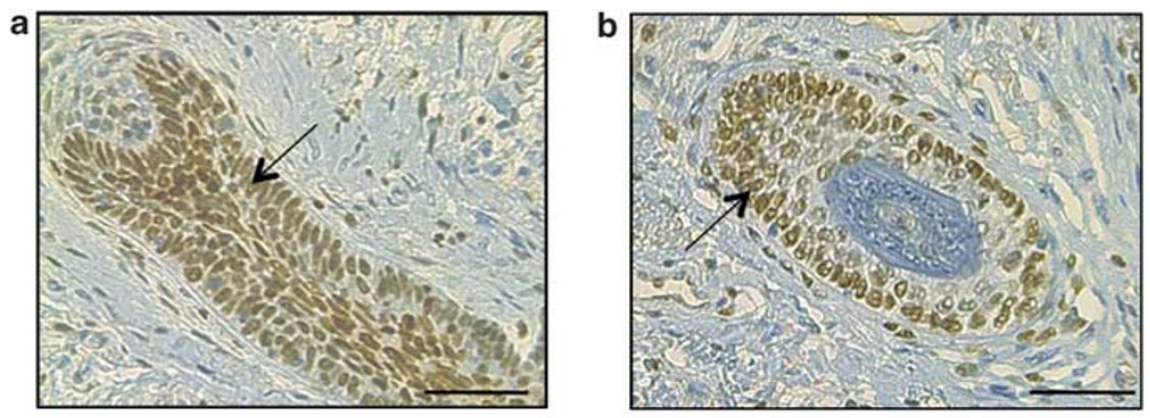

C
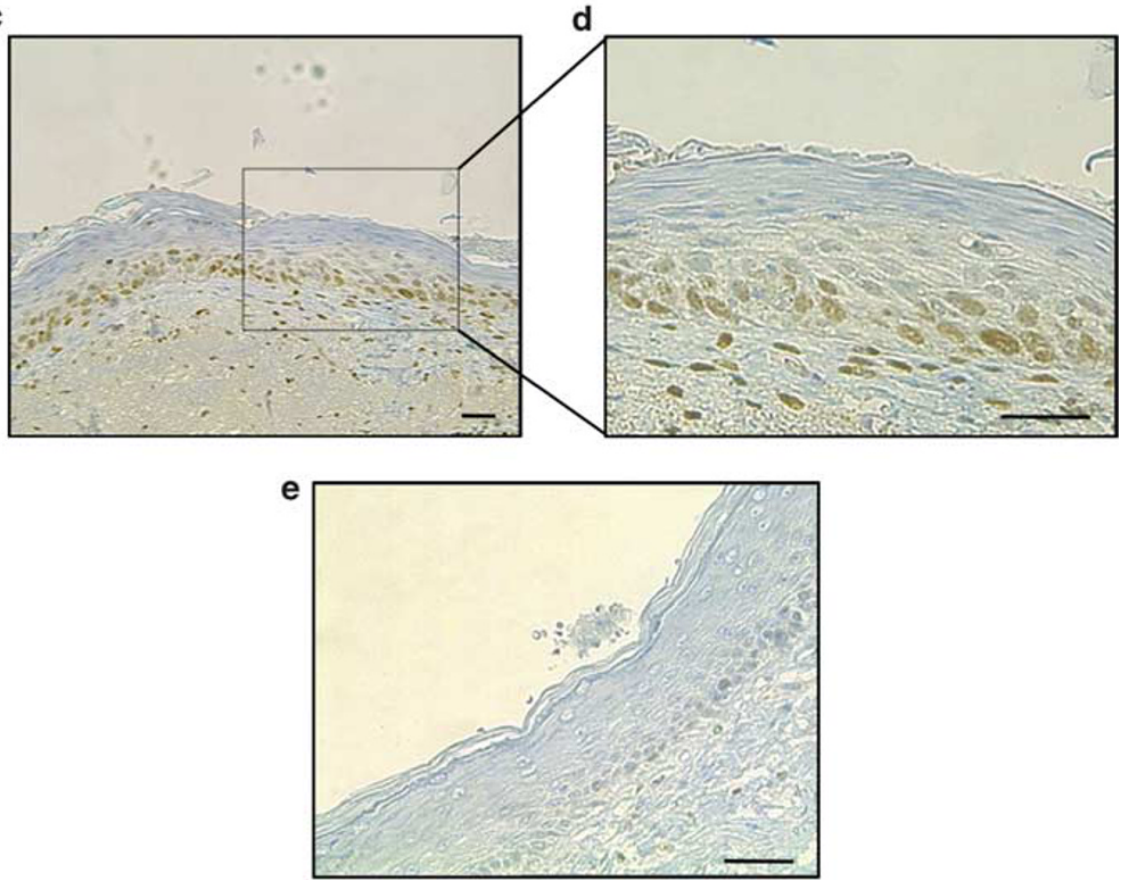

Figure 1 RUNX1 expression in human skin. Sections of paraffin-embedded normal human facial skin stained with anti-RUNX1 polyclonal (a-d) or control rabbit lgG (e) antibodies. The arrow shows RUNX1 nuclear labeling in longitudinal (a) and transversal (b) sections of a hair follicle. In the interfollicular epidermis (c and d), RUNX1 is essentially expressed in keratinocytes of the basal layer and its expression strongly decreases in suprabasal layers. The square in (c) represents the area of magnification shown in the (d). Scale bar $=50 \mu \mathrm{m}$ 
transition from proliferation to differentiation: in keratinocytes from three different donors, RUNX1 was strongly expressed in semi-confluent cells, then gradually declined when cells reached confluence and was weakly but reproducibly reactivated at the onset of differentiation. So, RUNX1 is expressed in human interfollicular epidermis and tightly regulated during the transition from proliferation to differentiation.

$\triangle$ Np63 modulates RUNX1 expression differentially in proliferative or differentiated human keratinocytes via two DNA regulatory sequences. RUNX1 has been recently identified by chromatin immunoprecipitation (ChIP) screens as a direct transcriptional target gene of $\Delta \mathrm{Np} 63$ in subconfluent mouse keratinocytes ${ }^{9}$ and HaCaT cell line. ${ }^{10,11}$ These studies revealed that $\triangle \mathrm{Np} 63$ binds an intronic region and one part of exon 1 of the $R U N X 1$ gene that are highly conserved between species, and that p63 is able to transactivate Runx1 expression. ChIP-quantitative PCR (QPCR) confirmed that RUNX1 exon 1 and intron 5 sequences are indeed significantly bound by p63 in HPK (Figure 3a).

The fact that p63 can bind two distinct regulatory DNA sequences of $R U N X 1$ gene suggested a more complex modulation of RUNX1 expression in human than that was previously proposed in mouse. ${ }^{9}$ Because RUNX1 expression is tightly regulated at the transition from proliferation to differentiation (Figures $2 \mathrm{e}$ and $\mathrm{f}$ ) and $\Delta \mathrm{Np} 63$ can exert separate control over keratinocyte proliferation and differentiation, ${ }^{4}$ we hypothesized that RUNX1 expression can be differentially modulated by $\Delta \mathrm{Np} 63$ in cells that stopped proliferation and began a differentiation program, versus still nonconfluent proliferating keratinocytes (Materials and Methods). RUNX1 expression was analyzed after $\Delta$ Np63specific inactivation by RNA interference in these humandifferentiated or proliferative keratinocytes. $\Delta \mathrm{Np} 63$ silencing was very efficient in both proliferating and differentiated keratinocytes at the mRNA (Figure 3b, left panel) and protein (Figure $3 \mathrm{c}$ ) levels. When $\Delta \mathrm{Np} 63$ was specifically knocked down in proliferative cells, RUNX1 expression was significantly decreased (Figure 3b right panel and $3 c$ left panel), suggesting that $\Delta$ Np63 positively regulates RUNX1 expression in proliferative human keratinocytes, as also observed in proliferative mouse keratinocytes. ${ }^{9}$ In contrast, reducing $\Delta$ Np63 expression in differentiated keratinocytes strongly increased RUNX1 expression (Figures $3 b$ and c, right panels), showing that $\Delta \mathrm{Np} 63$ negatively controls RUNX1 expression in

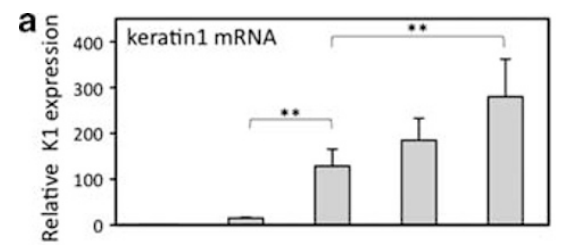

\section{e}
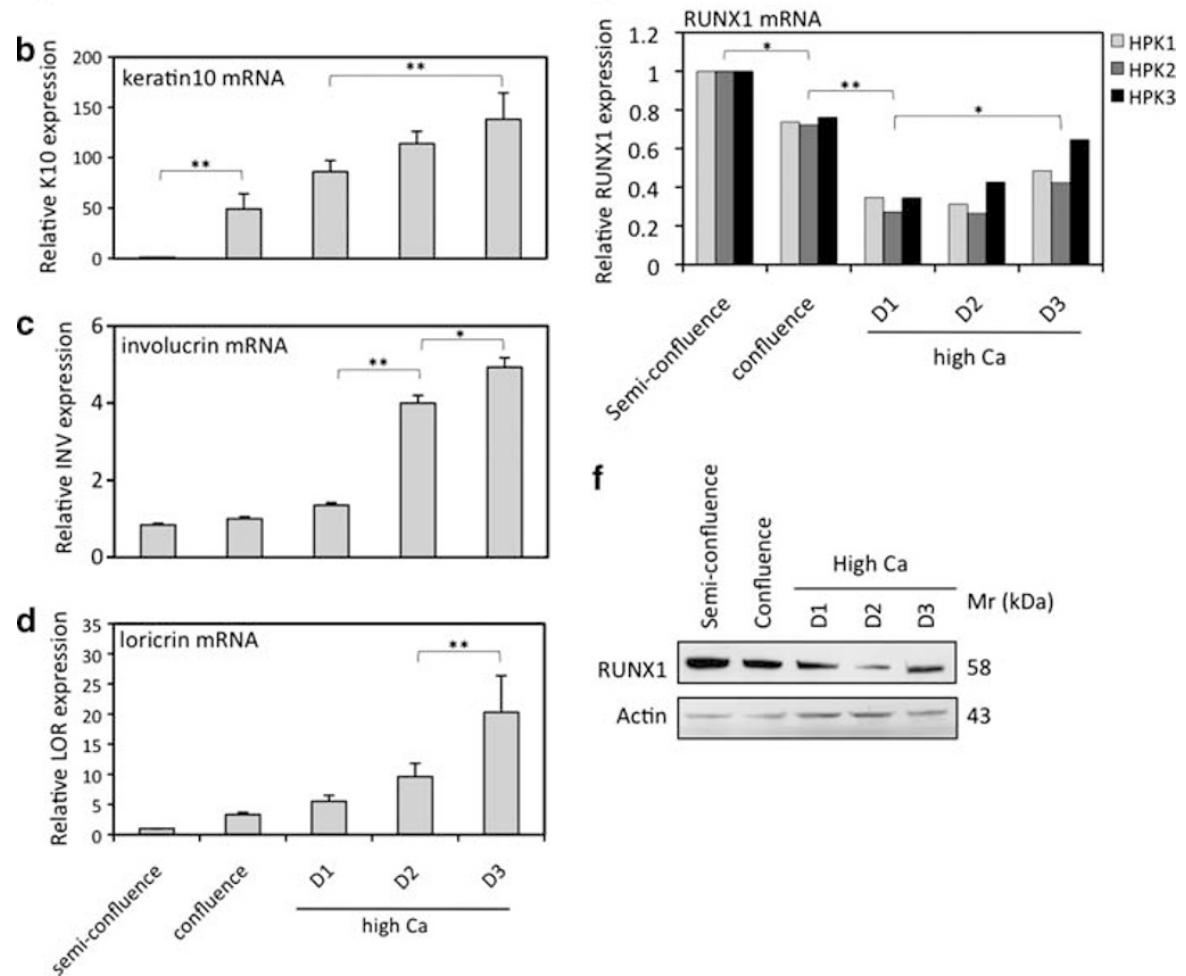

Figure 2 RUNX1 expression in cultured human keratinocytes. (a-e) Keratin1 (a), keratin10 (b), involucrin (c), loricrin (d) and RUNX1 (e) transcripts were quantified by QPCR in semi-confluent, confluent or $\mathrm{Ca}^{2+}$-induced differentiating keratinocyte cultures (D1, D2, D3) from three different donors (HPK1, HPK2, HPK3). Mean expression is indicated for $(\mathbf{a}-\mathbf{d})$; detailed expression for each culture is given for (e). (f) RUNX1 protein expression was analyzed from keratinocyte cultures established at the same stages 
cells that stopped proliferating and began differentiating. Taken together, these data showed that $\Delta$ Np63 differentially modulates RUNX1 expression depending on the proliferative or differentiation-committed state of human keratinocytes.

We then postulated that this differential regulation of RUNX1 expression could be linked to $\triangle N p 63$ differential binding onto $R U N X 1$ regulatory DNA sequences. ChIP-QPCR was therefore performed in HPK from three different donors in a proliferative or 3-day differentiated state. p63 was systematically bound (1.5-4-fold relative to the three donors) to the RUNX1 intron 5 sequence in both proliferative and differentiated keratinocytes (Figure $3 d$, left panel), but was able to bind exon 1 only in differentiated cells (1.6-2-fold; Figure 3d, right panel).

Overall, these results strongly suggest that $\Delta N p 63$ differentially regulates RUNX1 expression along the transition from proliferation to differentiation by differential binding of its regulatory sequences, highlighting the complexity of direct RUNX1 transcriptional regulation by $\Delta$ Np63 in human interfollicular keratinocytes. p53 binds the same RUNX1 regulatory sequences and cooperates with $\Delta \mathrm{Np} 63$ for regulation of RUNX1 expression. We postulated that other p53 family members, which are able in some specific cases to directly regulate the same transcriptional target genes, in either a similar or a converse way, ${ }^{21,22}$ could likewise modulate the $\Delta \mathrm{Np} 63$ action on RUNX1 regulation by binding its regulatory sequences. We therefore questioned whether the long p63 isoform, TAp63, as well as the p53 and/or p73 TFs could regulate RUNX1 expression in human interfollicular keratinocytes, given that they are expressed along the transition from proliferation to differentiation (data not shown). ${ }^{22}$ However, modulating TAp63 or p73 expression in primary keratinocytes did not affect RUNX1 expression levels (data not shown). In contrast, p53 was found to cooperate with $\Delta N p 63$ for RUNX1 regulation in HPK (Figure 4). Silencing of p53 alone in human keratinocytes had no effect on RUNX1 expression (Figure 4a). However, when both p53 and $\triangle$ Np63 expression was decreased by small interfering RNA (siRNA), RUNX1 expression was not repressed anymore
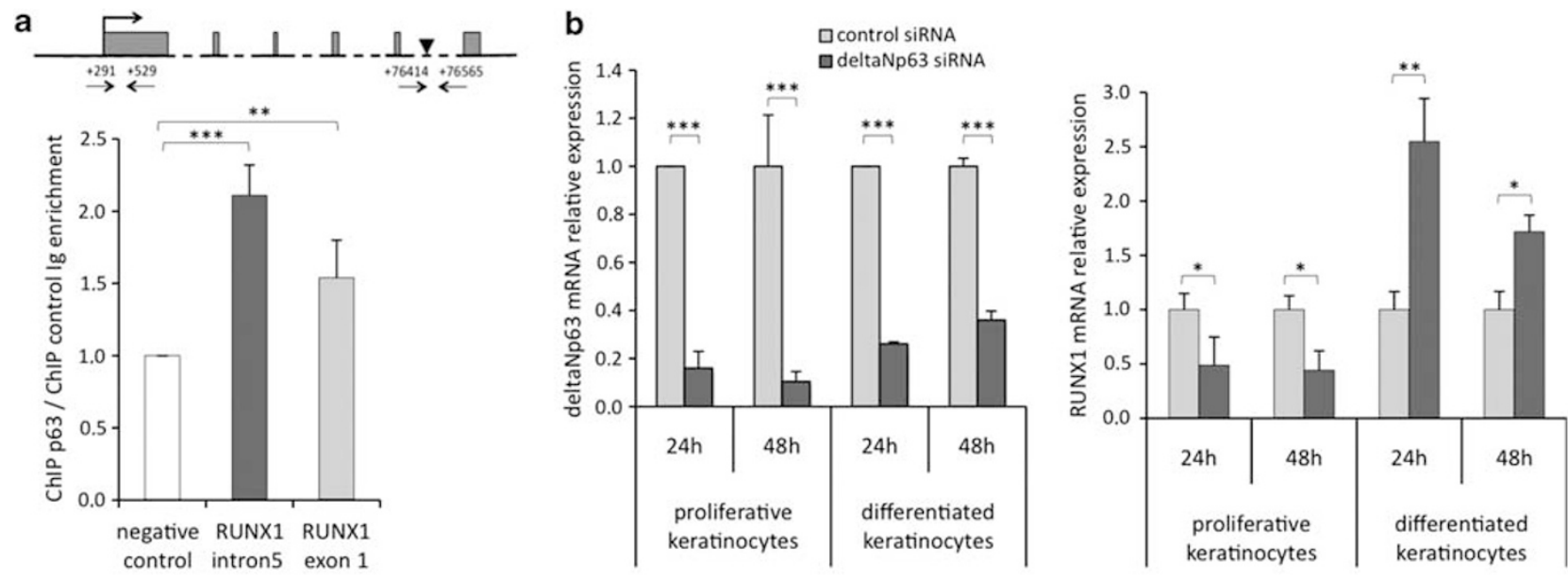

C
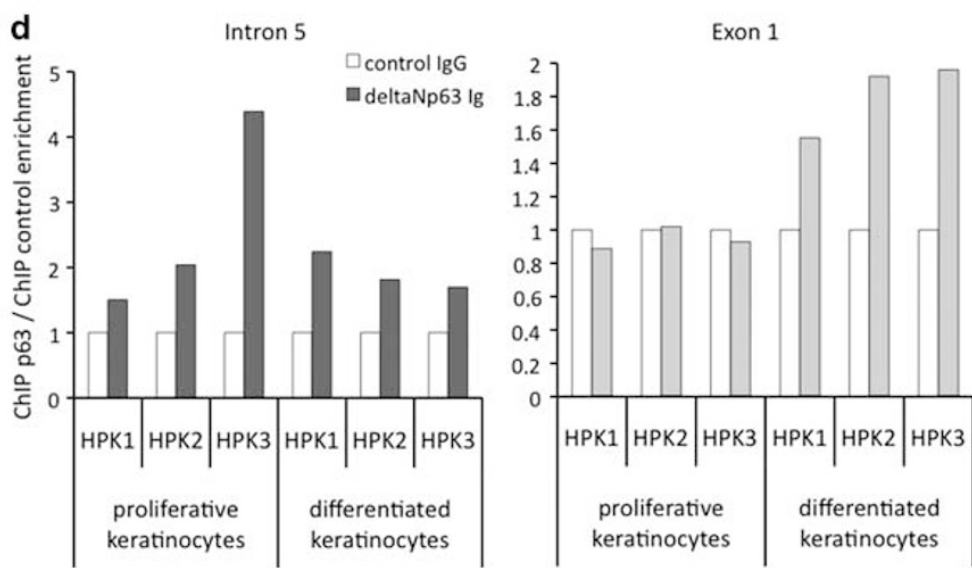

Figure $3 \quad \triangle N$ N63 can bind RUNX1 intron 5 and exon 1 DNA sequences and regulates its expression differentially in proliferating and differentiated keratinocytes. (a) Human RUNX1 gene map (upper panel) showing the exon-intron structure: arrows represent the DNA regions in exon 1 and intron 5 amplified by QPCR after ChIP experiments (lower panel). p63-ChIP assays were performed in three cultures of differentiated HPK. Enrichment of RUNX1 exon 1 and intron 5 regions was analyzed in comparison with a control promoter region. (b) $\triangle \mathrm{Np63}$ (left panel) and RUNX1 (right panel) transcript levels were quantified by RT-QPCR after treatment of proliferative or differentiated HPK with control or $\triangle \mathrm{Np63}$ siRNA. (c) p63 and RUNX1 protein levels were assessed by immunoblotting after treatment of proliferative (left panel) or differentiated (right panel) HPK with control or $\triangle N$ p63 siRNA. (d) p63-ChIP assays were performed in proliferative or 3-day differentiated HPK from three different donors (HPK1, HPK2, HPK3). Enrichment of the RUNX1 intron 5 (left panel) and exon 1 (right panel) regions was analyzed in comparison with a control promoter region by QPCR 
in proliferative keratinocytes (Figure $4 \mathrm{~b}$ left panel and $4 \mathrm{c}$ upper panel), compared with what was observed after $\Delta \mathrm{Np} 63$ inhibition alone (Figure $3 b$ right panel and $3 c$ left panel). So $\Delta$ Np63 needs p53 expression for RUNX1-positive regulation in proliferative keratinocytes. However, in differentiated keratinocytes, inhibition of both p53 and $\Delta$ Np63 did not disturb the $\Delta$ Np63 silencing-mediated effect on RUNX1 expression (compare Figure $4 \mathrm{~b}$ right panel with $3 \mathrm{~b}$ right panel and Figure $4 c$ lower panel with Figure $3 c$ right panel). These results are confirmed by $\Delta \mathrm{Np} 63$ silencing in the human HaCaT keratinocyte cell line (Figure 4d), known to express a mutated form of p53 that is transcriptionally inactive. ${ }^{23}$ Indeed, RUNX1 expression was not significantly modified in proliferative $\mathrm{HaCaT}$ cells when $\Delta \mathrm{Np} 63$ expression was decreased, conversely to what was observed in HPK that express a functional p53 protein (compare Figures $4 d$ with $3 b)$. These results were strengthened by studying RUNX1 expression profile in HaCaT cells: RUNX1 levels significantly decreased between proliferation and 1 day of differentiation but did not significantly increase again between 1 and 3 days of differentiation, conversely to what was observed in normal HPK (compare Figures $4 \mathrm{e}$ with $2 \mathrm{e}$ and $\mathrm{f}$ ). Thus, a functional $\mathrm{p} 53$ protein is necessary to properly regulate RUNX1 expression, in cooperation with $\Delta N p 63$, during the switch from proliferation to differentiation.

We confirmed by ChIP-QPCR that the p53 role in RUNX1 regulation depends on direct binding of p53 protein onto RUNX1 intron 5 and exon 1 (1.5-3.5-fold relative to the different donors; Figure 5a), whatever the state (proliferative or differentiated) of HPK.

To further investigate the specific role of each of the two DNA regulatory sequences of RUNX1 in $\triangle N p 63$ and p53 regulation, the parts of $R U N X 1$ exon 1 or intron 5 that are bound in vivo by these two TFs were individually cloned into a luciferase reporter vector. The $\mathrm{p} 53$ and $\Delta \mathrm{Np} 63$ overexpression effect (Figure 5b, left panel) on RUNX1 exon 1 and intron 5 transcriptional regulation was then analyzed by transactivation assays and revealed opposite transcriptional effects on the $R U N X 1$ gene; p53 overexpression significantly activated luciferase activity via $R U N X 1$ intron 5 without influencing exon 1 activity, whereas $\Delta$ Np63 overexpression did not affect intron 5 regulation but significantly repressed luciferase

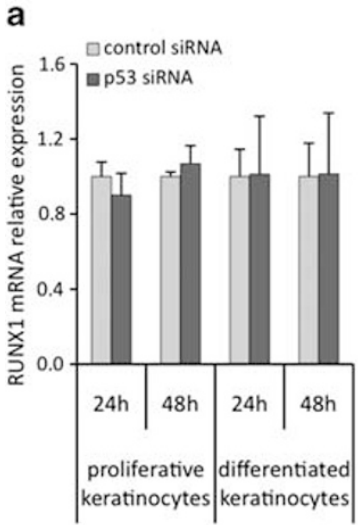

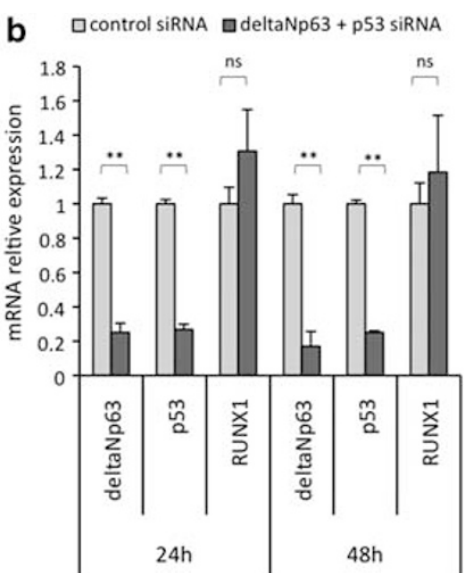

proliferative keratinocytes

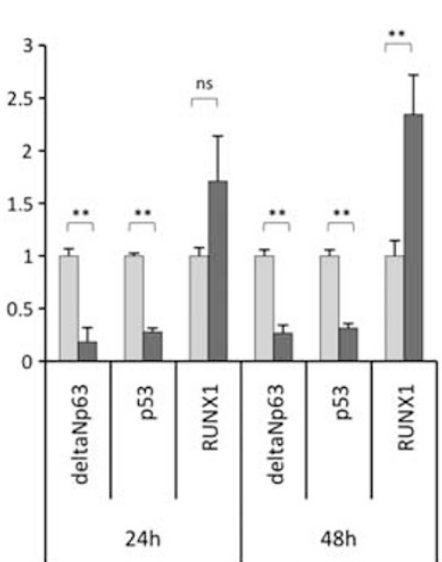

differentiated keratinocytes

e

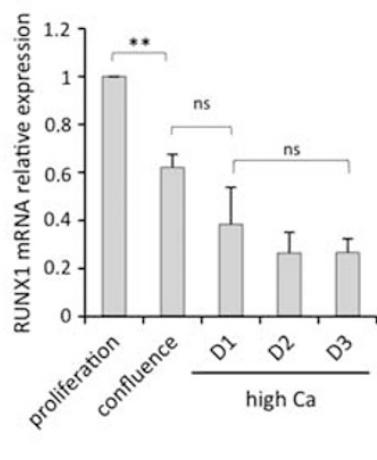

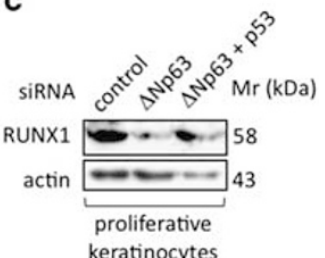

d

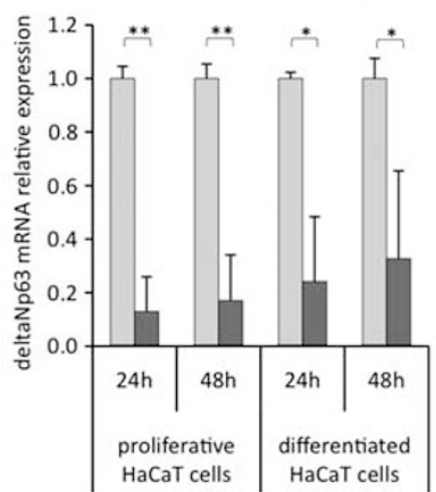

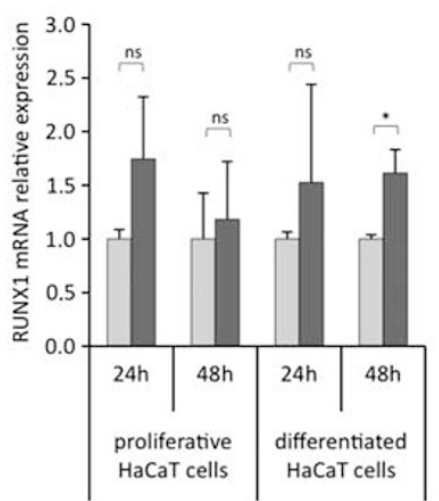

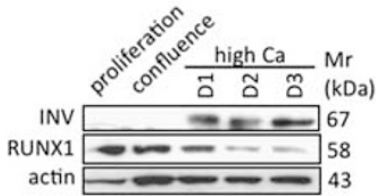

Figure 4 p53 cooperates with $\triangle N p 63$ to regulate RUNX1 in HPK. (a) RUNX1 transcript levels was quantified by RT-QPCR after treatment of proliferative or differentiated HPK with control or p53 siRNA. The effect of p53 siRNA on p53 expression was shown in Figure $2 b$. (b) p53, $\triangle$ Np63 and RUNX1 transcript levels were quantified by RT-QPCR after treatment of proliferative (left panel) or differentiated (right panel) HPK with control or p53 and $\triangle N p 63$ siRNA. (c) RUNX1 protein level was assessed by WB $48 \mathrm{~h}$ after treatment of proliferative (upper panel) or differentiated (lower panel) HPK with both p53 and $\triangle N p 63$ siRNA. (d) $\triangle N p 63$ (left panel) and RUNX1 (right panel) transcripts levels were quantified by RT-QPCR after treatment of proliferative or differentiated HaCaT cells with control or $\triangle$ Np63 siRNA. (e) RUNX1 transcripts were measured by QPCR at different stages of the HaCaT cell cultures (left panel). Involucrin (INV) and RUNX1 proteins expression was assessed by immunoblotting at different stages of the HaCaT culture (right panel) 

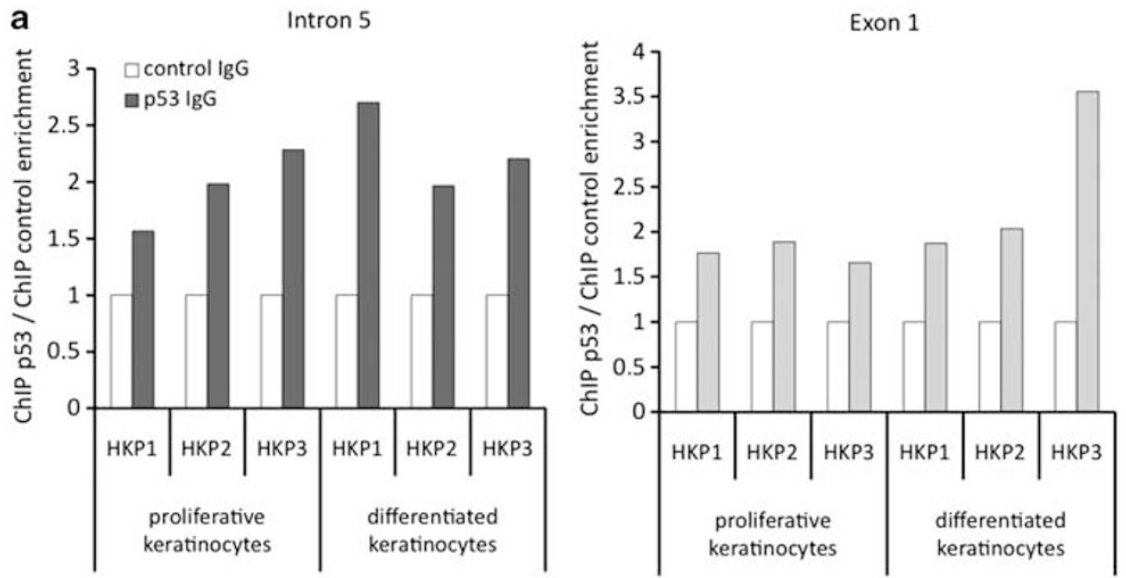

b
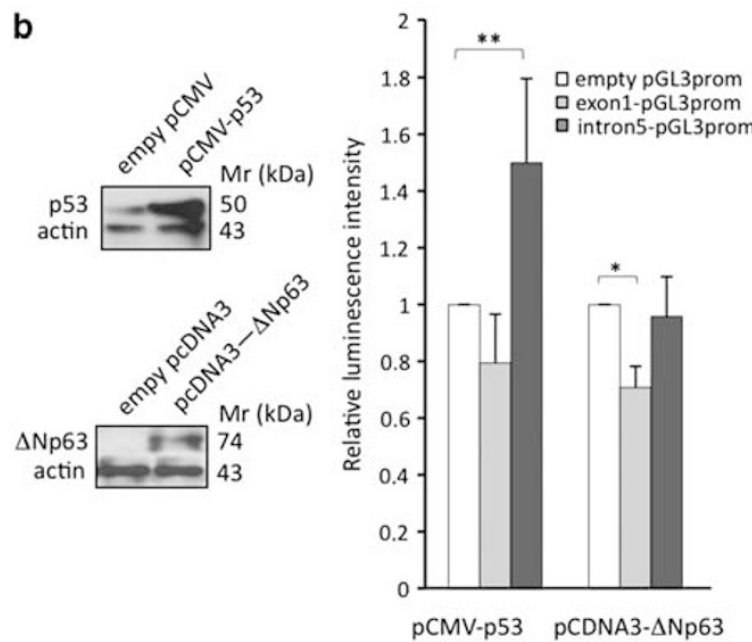

C

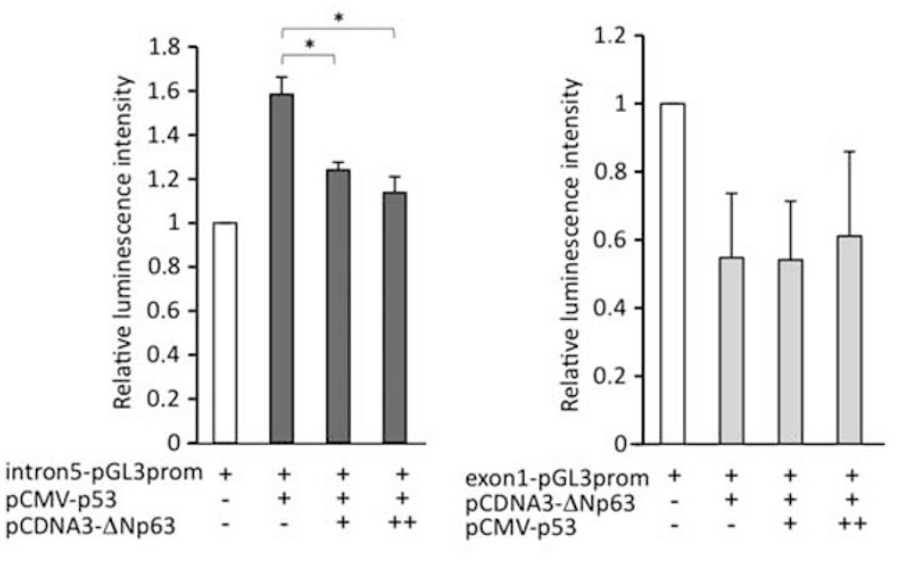

Figure 5 Functional interplay between $\triangle \mathrm{Np63}$ and p53 to regulate RUNX1 expression. (a) p53-ChIP assays were performed in proliferative or 3-day differentiated HPK from three different donors (HPK1, HPK2, HPK3). Enrichment of the RUNX1 intron 5 (left panel) and exon 1 (right panel) regions was analyzed in comparison with a control promoter region by QPCR. (b) p53 and $\triangle$ Np63 (left panels) protein levels were analyzed by immunoblotting after $48 \mathrm{~h}$ transfection of pCMV-p53 and pcDNA3- $\Delta$ Np63 vectors or their respective empty vectors in HEK293 cells. Results shown are representative of two independent experiments. The right panel shows reporter gene assays for part of exon 1 and intron 5 of RUNX1 cloned in pGL3 promoter after overexpression of p53 or $\Delta$ Np63 proteins. Four independent experiments were performed. (c) Competition luciferase assays were performed with increasing concentration (200 or $400 \mathrm{ng}$ ) of pcDNA3-p63 for competition with p53 on RUNX1 intron 5 (left panel) and with increasing concentration (200 or $400 \mathrm{ng}$ ) of pCMV-p53 for competition with $\triangle \mathrm{Np63}$ on RUNX1 exon 1 (right panel)

activity via the RUNX1 exon 1 (Figure $5 \mathrm{~b}$, right panel). Moreover, additional experiments showed that $\triangle \mathrm{Np63}$ was able to compete with p53 on RUNX1 intron 5 (Figure 5c), in agreement with in vivo ChIP experiments. These data suggested that $\triangle \mathrm{Np} 63$ could repress RUNX1 expression by binding to its exon 1 regulatory sequence, whereas p53 could induce RUNX1 expression by binding to its intron 5 DNA sequence.

RUNX1 modulates keratinocyte proliferation and directly regulates $K R T 1$ gene expression. As $\Delta \mathrm{Np} 63$ has a critical function in the regulation of entry into differentiation and is involved in RUNX1 coregulation along with p53, we investigated whether RUNX1 may also have a key role in the regulation of proliferation and differentiation processes in human interfollicular epidermis.

To this end, the RUNX1 role in proliferation was explored in proliferating HPK. RUNX1 overexpression under exponential growth conditions led to a strong increase in mRNA (data not shown) and protein RUNX1 levels (Figure 6a, left panel) associated with a significant decrease in cell number compared with an empty vector control (Figure 6a, right panel). Conversely, RUNX1 knockdown (Figure 6b, left panel), by transfecting a validated specific siRNA, ${ }^{24}$ significantly increased cell number (Figure $6 \mathrm{~b}$, right panel). This modification of cell number is due to a higher proliferation rate (Figure 6c). In fact, RUNX1 inhibition by siRNA increased the percentage of BrdU-positive keratinocytes (R2 region) and concomitantly decreased the percentage of keratinocytes in the $\mathrm{G} 1$ phase (R1 region) (Figure $6 \mathrm{~d}$, left panel). This significant RUNX1 effect on cell cycle modulation was consistently found in four independent experiments using keratinocytes from various donors (Figure 6d, right panel). Interestingly, we found that RUNX1 depletion by siRNA led to a significant decrease of $p 21$ expression, a negative regulator of cell cycle, as well as to a significant increase of proliferation 

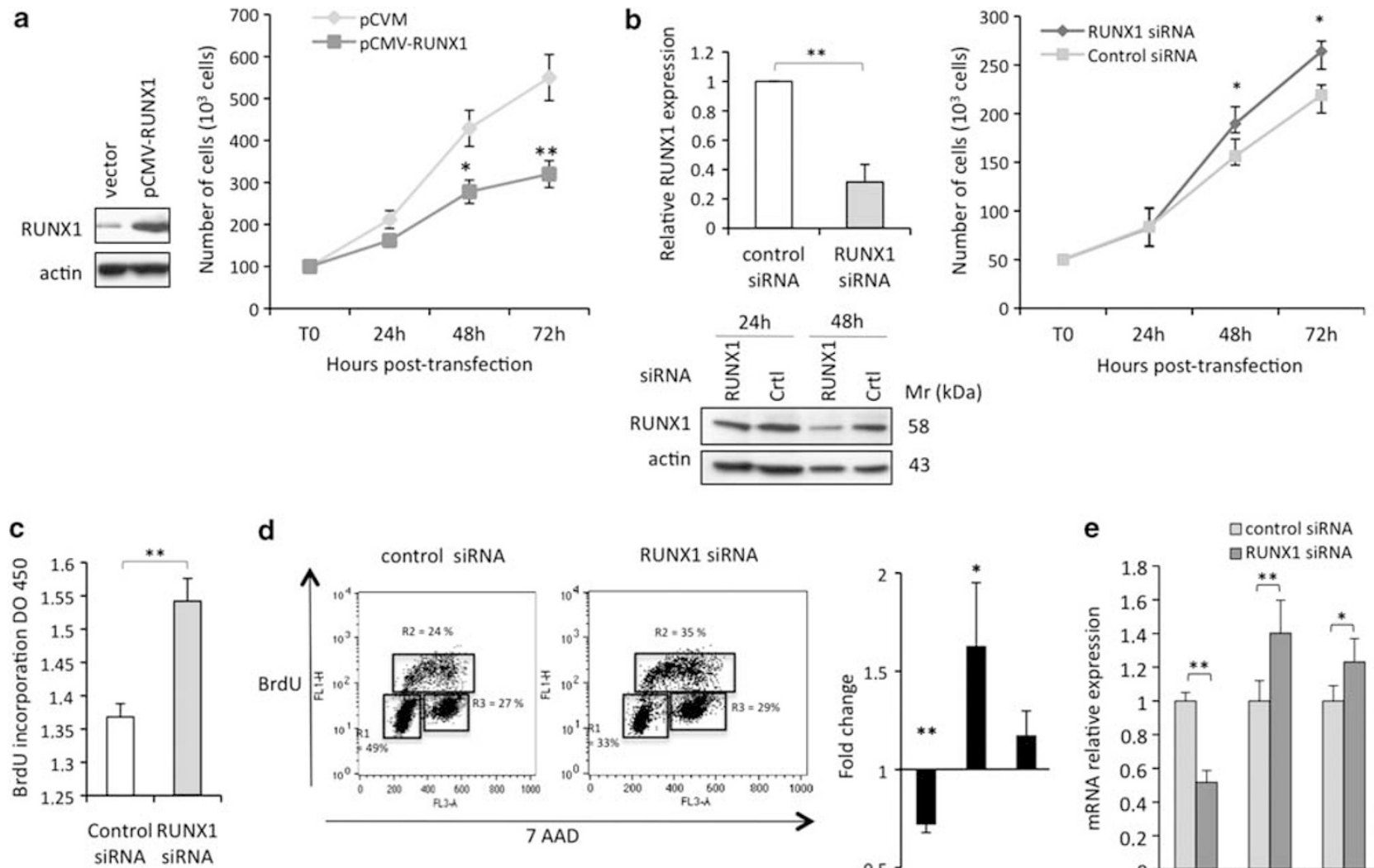

d

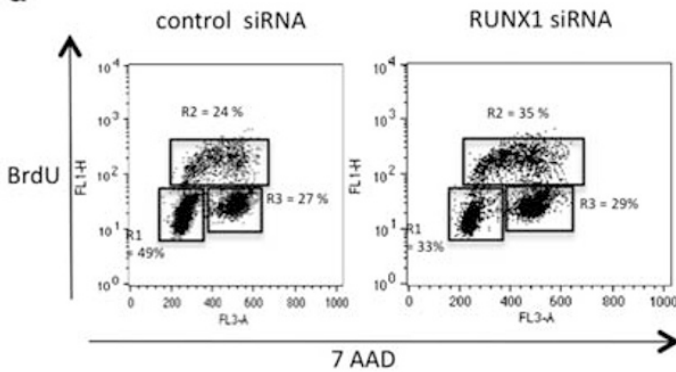

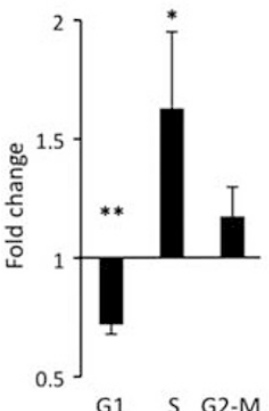

e

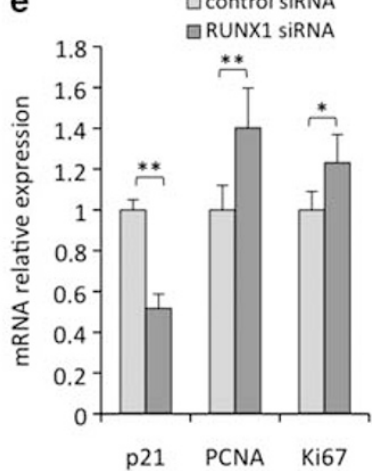

Figure 6 RUNX1 represses human keratinocyte proliferation. (a) RUNX1 overexpression using pCMV-RUNX1 was verified by western blotting (left panel) compared with an empty vector and cell numbers (right panel) were determined. Error bars are mean $+/-$ S.D. of triplicate samples from one representative experiment. (b) RUNX1 mRNA (upper left panel) and protein (lower left panel) levels were measured after RUNX1 silencing by an siRNA targeting RUNX1 versus a control non-targeting siRNA in proliferating keratinocytes and cell numbers were determined (right panel). (c) Proliferation study using BrdU incorporation assay was performed in triplicate in three independent experiments with different donors. (d) Cell cycle analysis of siRUNX1-transfected keratinocytes shows percentages of cell cycle phases $(R 1=G 0 / G 1, R 2=S, R 3=G 2 / M$, left panel). Dot plots illustrate one representative experiment. The graph (right panel) shows the percentage of stained RUNX1 siRNA-treated keratinocytes/ the percentage of stained control siRNA-treated keratinocytes. (e) p21, PCNA and Ki67 transcripts levels were quantified by RT-QPCR $24 \mathrm{~h}$ after treatment of proliferating primary keratinocytes with control or RUNX1 siRNA

cell nuclear antigen (PCNA) and Ki67, two well-known markers of proliferation (Figure 6e). Taken together, these data indicate that RUNX1 is a negative regulator of cell proliferation in cultured human keratinocytes.

We also investigated whether RUNX1 has a role in regulating the early differentiation process, as RUNX1 and its regulator $\triangle \mathrm{Np} 63$ are both expressed in the first differentiated layers of the human epidermis. A bioinformatic screen for RUNX1 binding sites in promoter regions of 27 genes selected for their strong expression in human granular and spinous keratinocytes ${ }^{25}$ revealed highly conserved RUNX1 binding sites (score $>0.9$ ) in the promoter sequences of 3 genes. One site in the promoter $(-577$ to -563$)$ and another in the first exon $(+186$ to +190$)$ was identified for KRT1 (encoding keratin1), whereas TGM1 and LOR (respectively encoding transglutaminase 1 and loricrin) exhibited only one site ( -508 to -494 and -323 to -309 , respectively). As the presence of a consensus binding site is merely suggestive of a possible regulation, RUNX1 depletion was conducted by siRNA transfection. It led to reduction of KRT1 and LOR mRNA levels by $>30 \%$ whereas TGM1 mRNA was not significantly modified (Figure 7a), showing that KRT1 and LOR are RUNX1 downstream targets in human keratinocytes. To investigate whether RUNX1 directly regulates KRT1 and LOR expression, the effect of RUNX1 and/or its cofactor $\operatorname{CBF} \beta$ on the promoter regulation of these two genes was examined by luciferase assay (Figure $7 b$ ). RUNX1 overexpression did not modify the levels of LOR reporter activity, whereas it reproducibly and significantly increased KRT1 reporter activity. When one of the two potential RUNX1 binding sites was deleted, basal luminescence intensity and induced luciferase activity after RUNX1 and/or CBF $\beta$ transfection were abolished. Taken together, these findings indicate that RUNX1 positively regulates KRT1 expression by directly binding to its promoter. Overexpression or inhibition of RUNX1 in HPK led to a correlated regulation of endogenous KRT1 protein (Figure 7c), confirming the positive regulation exerted by RUNX1 on KRT1 protein. Finally, ChIP-QPCR experiments using three different anti-RUNX1 antibodies showed strongly enriched KRT1 promoter sequences in the immunoprecipitated fraction, (3- to 13-fold) whereas $L O R$ and TGM1 target sequences were not enriched 


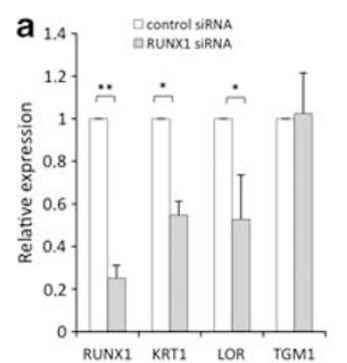

C

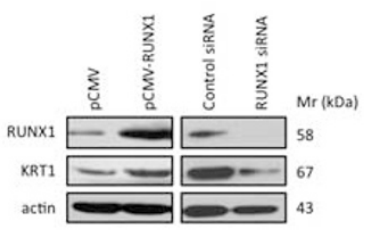

b
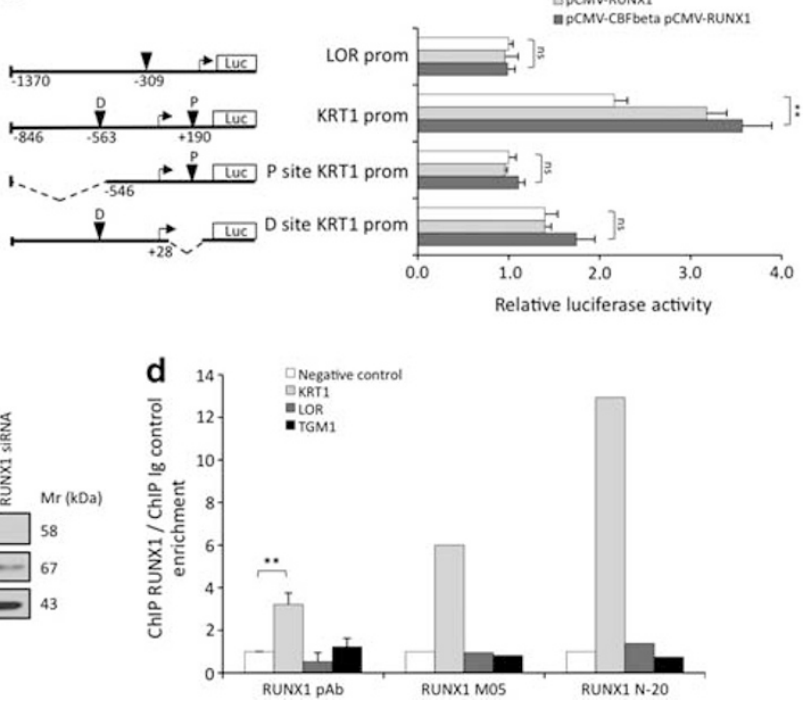

Figure 7 RUNX1 directly regulates KRT1 expression. (a) RUNX1, KRT1, LOR and TGM1 transcripts were assessed by RT-QPCR $24 \mathrm{~h}$ after transfection by RUNX1 or control siRNA. (b) Reporter gene assays were done for LOR promoter and full or partially deleted KRT1 promoters. (c) RUNX1 and KRT1 expression was assessed by western blotting $48 \mathrm{~h}$ after pCMV-RUNX1 or RUNX1 siRNA transfection in primary keratinocytes. (d) ChIP-QPCR assays using various anti-RUNX1 antibodies or control Ig showed the enrichment of KRT1, LOR, TGM1 promoter and control intragenic sequence. For RUNX1 pAb AB1, results are means $+/-S . D$. from three independent experiments

(Figure 7d), in agreement with the above expression analysis and reporter assay data. Overall, these results demonstrate that, in cultured human keratinocytes, RUNX1 directly binds the KRT1 promoter sequence and validate KRT1 as a direct RUNX1 transcriptional target in these cells.

Taken together, these data showed that RUNX1, tightly regulated by $\Delta \mathrm{Np} 63$ and p53 during the transition from proliferation to differentiation in human interfollicular keratinocytes, is able to modulate both the proliferation process and the expression of early differentiation markers.

RUNX1 expression is dysregulated in human skin carcinomas. BCCs and SCCs are the two commonest nonmelanoma cancers of epithelial origin in the skin. BCCs are derived from or closely related to long-term resident progenitor cells of the interfollicular epidermis, ${ }^{26}$ whereas SCCs might originate from both hair follicles and interfollicular epidermis. ${ }^{27}$ Missense mutations in the tumor suppressor gene p53 are a common genetic feature of these tumors, as a result of a complex sequence of events initiated by exposure to UV light. ${ }^{28}$ BCCs and SCCs are also characterized by $\Delta$ Np63 protein upregulation. ${ }^{7,29}$ As RUNX1 expression is tightly regulated by differential interplay between p53 and $\Delta$ Np63, we analyzed RUNX1 expression by immunochemistry in a panel of BCCs and SCCs (Figure 8). All BCCs overexpressed RUNX1, compared with RUNX1 expression level in the basal layer of surrounding epidermis (Figure 8a), but to varying degrees: $3 / 18$ showed low RUNX1 expression with $<10 \%$ immunostained cells, $6 / 18$ had a moderate immunostaining with $40-80 \%$ positive cells, and 9/18 showed a high RUNX1 expression with over $80 \%$ immunostained cells (Figure $8 \mathrm{c}$ ). By contrast, only 9 out of 15 SCCs presented RUNX1 immunostaining (Figures $8 \mathrm{~b}$ and $\mathrm{c}$ ). The majority exhibited a low to moderate RUNX1 staining.
In conclusion, these data support the contention that RUNX1 is dysregulated in nonmelanoma skin tumors and is expressed at higher levels in BCCs than in SCCs.

\section{Discussion}

The present study shows for the first time that RUNX1 is expressed in human interfollicular epidermis, while being restricted to hair follicles in mouse skin. ${ }^{18}$ These results are in line with the strong $\Delta \mathrm{Np} 63$ expression in human interfollicular epidermis, shown as a direct transcriptional regulator of RUNX1. ${ }^{9-11}$ We have also demonstrated that RUNX1 is tightly regulated by a complex mechanism involving $\Delta \mathrm{Np63}$ and $\mathrm{p} 53$, and control cell proliferation and onset of differentiation.

Among the members of the p53/p63/p73 TFs family, TAp63 and the short $(\Delta N)$ and long $(T A)$ isoforms of $p 73$ are also expressed in human interfollicular keratinocytes. ${ }^{22}$ However, the $\Delta$ Np63 isoform was shown as the main p63 mediator regulating the switch from proliferation to differentiation, whereas several studies involved TAp63 and p73 isoforms in the development of the granular and horny layers. ${ }^{1,4,30,31}$ These data agree with our results, suggesting that TAp63 and p73 are dispensable for RUNX1 expression in human interfollicular epidermis.

Only p53 cooperates with $\Delta$ Np63 during the transition from proliferation to differentiation of human interfollicular keratinocytes. $\Delta \mathrm{Np} 63$ would have a key role for the entry into differentiation by inhibiting RUNX1 expression, whereas, in proliferating keratinocytes, p53 would overcome the $\Delta$ Np63negative regulatory effect to activate RUNX1. Our results reinforce and complete the study conducted in human keratinocytes by Truong et al. ${ }^{4}$ that clearly demonstrated a role for p53 in epidermal proliferation but not in the 
a
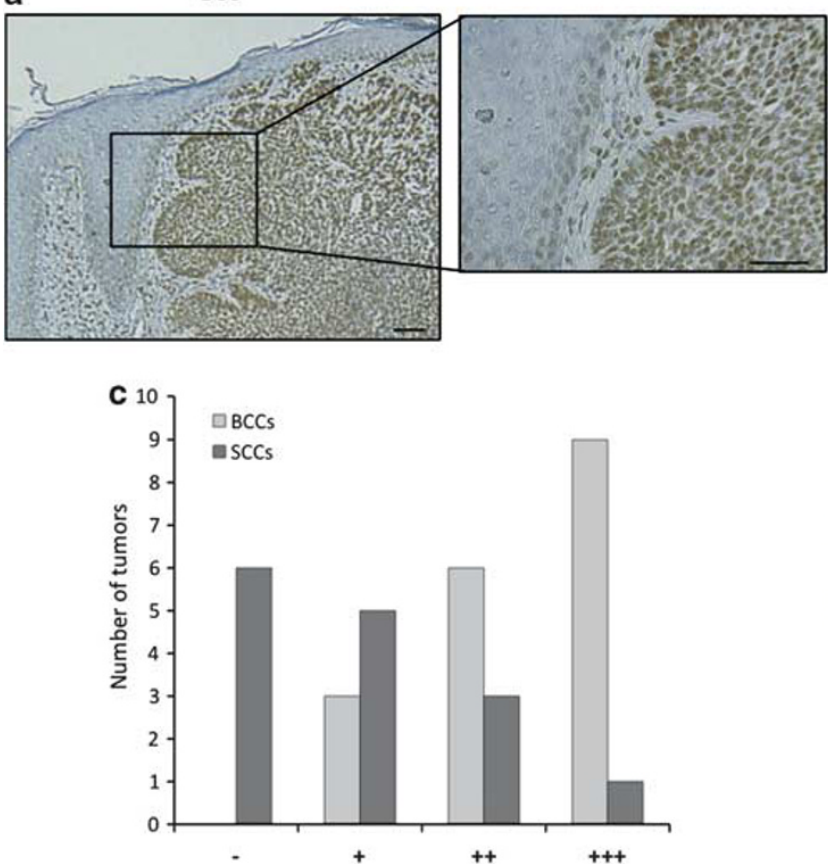

scc
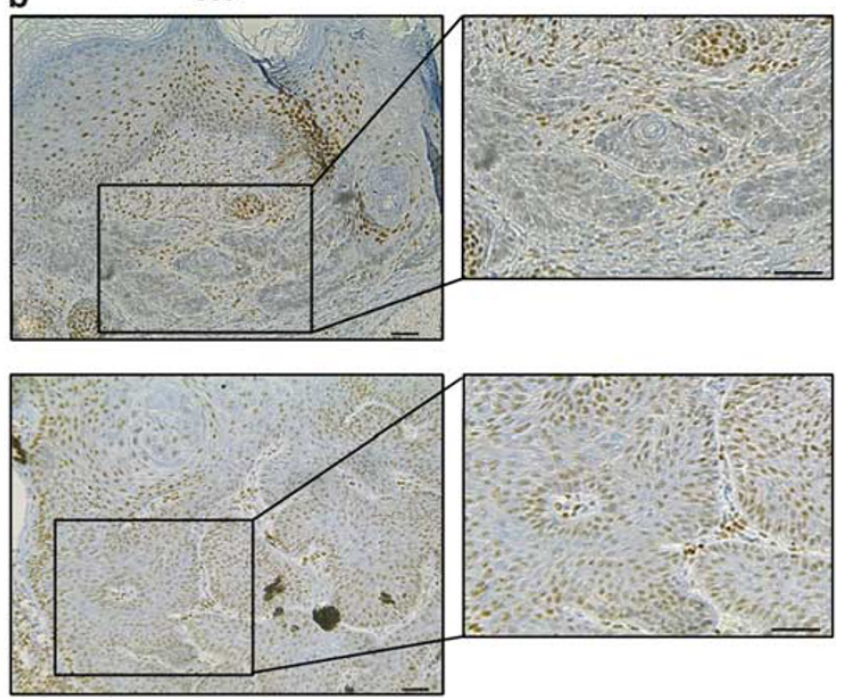

Figure 8 RUNX1 expression is deregulated in BCCs and SCCs. (a and $\mathbf{b})$ Sections of paraffin-embedded specimens from human BCCs (a) and SCCs (b) were stained with an anti-RUNX1 polyclonal antibody. The squares represent the areas of magnification containing the tumors shown in the inset. For the BCC section (a), the magnification shows a higher RUNX1 staining in the tumor than in the basal layer of the surrounded epidermis. For the SCC section (b), the upper panel shows a negative specimen for RUNX1 staining and the lower panel shows a positive one. Scale bar $=50 \mu \mathrm{m}$. (c) RUNX1 expression is semiquantitatively assessed in 18 BCCs and 15 SCCs; $-:$ no staining; + : low, ++ : moderate, +++ : strong staining

differentiation process. Moreover, the importance of p53 for regulation by $\mathrm{p} 63$ of another TF, Krueppel-like factor 4 (KLF4), was recently shown in proliferative epidermal cells. ${ }^{32}$ Collectively, our data of expression, ChIP and reporter assays allows us to propose a model for the regulation of RUNX1 expression: in proliferating keratinocytes, both $\triangle \mathrm{Np} 63$ and p53 would bind RUNX1 intron 5, that in turn leads to RUNX1 activation by p53 via this positive regulation element. In keratinocytes that reach confluence, stop proliferating and begin differentiating, $\Delta N p 63$ would join p53 onto RUNX1 exon 1 regulatory sequence that has for consequence RUNX1 repression by $\Delta \mathrm{Np63}$. Therefore, the tight control of RUNX1 expression by this functional interplay between the two TFs $\Delta \mathrm{Np63}$ and p53 could lead to a fine regulation of downstream molecular events to allow a proper transition from proliferation to differentiation in human interfollicular keratinocytes.

Indeed, we showed that the tight regulation of RUNX1 expression leads to the regulation of both proliferation and early differentiation. RUNX1 inhibits human interfollicular keratinocyte proliferation. This antiproliferative effect seems to be specific to this cell type, as RUNX1 is known to be a positive regulator of G1-to-S cell cycle progression in hematopoietic tissue, ${ }^{14}$ neural progenitors ${ }^{16}$ and, more recently, in mouse hair follicle stem cells. ${ }^{17}$ However, a strong correlation between negative cell cycle regulators and RUNX1 expression was observed in endometrial carcinoma, ${ }^{33}$ underlying the complexity of RUNX1 cell growth regulation, in line with the fact that RUNX1 can act as both a transcriptional repressor and activator. We also showed that RUNX1 could activate the $K R T 1$ gene in human keratinocytes through direct binding to its promoter region. KRT1 induction is one of the earliest events in keratinocyte differentiation, as it occurs in basal keratinocytes that have ceased mitotic activity. ${ }^{34}$ These results are even more interesting as $\Delta \mathrm{Np} 63$ is also responsible for KRT1 regulation via c-Jun $\mathrm{N}$-terminal kinase/AP1 activation. ${ }^{35}$ Thus, RUNX1 appears to have a key role together with $\Delta$ Np63 in the switch from proliferation to differentiation in the basal layer, by downregulating the cell cycle and inducing early differentiation markers such as KRT1.

Finally, we showed that RUNX1 expression is dysregulated in the two most widespread nonmelanoma skin cancers of epithelial origin, BCCs that are locally destructive but metastasizes only exceptionally, and SCCs with high metastatic potential. ${ }^{36}$ RUNX1 seems to be globally more expressed in BCC cells, which are phenotypically closed to basal proliferating keratinocytes, than in SCC cells, which appear as more differentiated. This RUNX1 pattern is not identical with that of $\Delta \mathrm{Np} 63$ expression, although $\Delta \mathrm{Np} 63$ is also more strongly expressed in BCCs compared with SCCs (data not shown; ${ }^{7,29,32}$ ). Conversely, our data and those of the literature show a better correlation between RUNX1 and p53 expression. As p53 is mutated and highly expressed in SCCs (whereas expression is lower in $\mathrm{BCCs}^{29,32}$ ), it could lead to a lack of RUNX1 activation in differentiated SCC cells, which in turn would be silenced by $\Delta N p 63$ overexpression, according to our proposed model. This model is strengthened by the fact that clusters of cells overexpressing a mutated form of $p 53$, considered as potential precursors of SCCs, can be detected in the interfollicular epidermis before the skin tumors arise; ${ }^{37}$ and that the negative regulation of KLF4, another target of p63, is subverted by p53 mutations commonly found in SCC. ${ }^{32}$ The fact that only part of SCCs are positive for RUNX1 
expression suggest that it may reflect varying carcinogenic insults and may be linked in particular with differential p53 mutations.

In summary, we present evidence that RUNX1 TF expression is regulated by a functional interplay between $\Delta N p 63$ and p53, and that RUNX1 is involved in the control of the transition between proliferation and early differentiation in human keratinocytes. The use of large-scale genomic approaches such as ChIP-chip or ChIP-seq would be of great interest for identifying novel RUNX1 target genes in proliferating versus differentiated keratinocytes in order to elucidate its role in the regulation of epidermal homeostasis.

\section{Materials and Methods}

Cell cultures. HPK, isolated from neonatal foreskin, are cultured in KGM2 medium (PromoCell, Heidelberg, Germany) as previously described. ${ }^{38}$ This study was approved by the ethical research committee 'Comité de Protection des Personnes Sud-Est-Il' (CODECOH Number DC-2008-262). A written informed consent was obtained from infants' parents according to the French bioethical law of 2004 (loi 94-654, 29/07/1994) and the guidelines of the Helsinki Declaration. HEK293 cells (provided by Pr. Arrigo, CNRS, Lyon), were cultured in DMEM 10\% (V/V) fetal bovine serum (Lonza). Differentiation of HPK or HaCaT cells was induced by a shift to high calcium concentration $(1.8 \mathrm{mM})$ after culture at low $\mathrm{Ca}^{2+}$ concentration $(0.06 \mathrm{mM})$ up to confluence. To analyze the proliferating state, keratinocytes were transfected at $40 \%$ of confluency and collected before confluency. To study the differentiating state, keratinocytes were transfected at $90 \%$ of confluency and collected at the beginning of differentiation.

Transient transfection. RUNX1 was overexpressed using JetPei transfection (PolyPlus, Illkirch, France) with $1 \mu \mathrm{g}$ of pCiNeo-RUNX1 or pCMVRUNX1 (provided by Dr. Mouchiroud, CNRS, Lyon) following the manufacturer's instructions. For silencing experiments, keratinocytes were transfected at $20 \mathrm{nM}$ final concentration using Hyperfect transfection reagent (Qiagen, Germantown, $M D, U S A)$ according to the manufacturer's directions. siRNA sequences are listed in Supplementary Table 1.

Real-time RT-PCR. Total RNA was extracted with the RNAeasy mini-kit (Qiagen), reverse-transcribed into cDNA by MuLV reverse-transcriptase (Fermentas, Waltham, MA, USA) and analyzed by real-time PCR using the Absolute Blue QPCR SYBRGreen Rox mix (ThermoScientific, Waltham, MA, USA) on a MX3000P real-time PCR system (Stratagene, Santa Clara, CA, USA) with the primers shown in Supplementary Table 2. Results were obtained from independent experiments using keratinocytes from three different donors run in triplicates and were normalized to the 18S rRNA expression level.

Western blot, immunofluorescence and immunohistochemistry. Protein extraction and blotting were performed as described. ${ }^{39}$ Actin was used as a loading control. Results were obtained from three independent experiments with different donors. Immunohistochemistry was done as previously described. ${ }^{38}$ Antibodies are listed in Supplementary Table 3.

Proliferation assay and cell cycle analysis. For proliferation assay, BrdU was added or not to the medium. The incorporated BrdU amount was determined $6 \mathrm{~h}$ later by the Cell Proliferation ELISA BrdU colorimetric assay (Millipore, Darmstadt, Germany) according to the manufacturer's instructions using a plate reader (Victor3, Perkin Elmer, Waltham, MA, USA). The proliferation extent was expressed as OD mean values in the presence of BrdU$\mathrm{OD}$ mean values without $\mathrm{BrdU} \pm$ S.D. of triplicate wells. Keratinocyte cell cycle progress was examined $24 \mathrm{~h}$ after RUNX1 or control siRNA transfection using FITC-BrdU/7-aminoactinomycin D Flow Kit (BD Pharmingen, Franklin Lakes, NJ, USA) according to the manufacturer's protocol. Fluorescence analysis was performed on a FACS Calibur using Cellquest software (BD Biosciences, Franklin Lakes, NJ, USA).

Chromatin immunoprecipitation. Equivalent numbers of HPK in a proliferative or 3-day differentiated state were lysed after crosslinking with $1 \%$ formaldehyde (Sigma, St. Louis, MO, USA). Chromatin was sheared by sonication, precleared with proteinG-agarose/salmon sperm DNA beads (Upstate, Darmstadt, Germany) and control immunoglobulins (Zymed, South San Francisco, CA, USA), incubated overnight at $4{ }^{\circ} \mathrm{C}$ with $2 \mu \mathrm{g}$ anti-p63 $4 \mathrm{a} 4$ (sc-8431 Santa Cruz, Santa Cruz, CA, USA), anti-p53 DO-1 (sc-126 Santa Cruz) or control mouse immunoglobulins. Immune complexes were collected with proteinG-agarose/ salmon sperm DNA beads, washed, extracted in $1 \% \mathrm{SDS}, 0.1 \mathrm{M} \mathrm{NaHCO}_{3}$ and treated with RNAse $A$ and proteinase $\mathrm{K}$. Protein crosslink was reverted by heating at $65{ }^{\circ} \mathrm{C}$ for at least $5 \mathrm{~h}$. DNA was purified using Qiaquick PCR-Purification Kit (Qiagen). QPCR were performed on the immunoprecipitated genomic DNA by using specific primers listed in Supplementary Table 4.

Promoter analysis. $5^{\prime}$ regulatory sequences $(-750,+250$ from TSS) were retrieved from the promoter database at http://rulai.cshl.edu/cgi-bin/TRED/ and screened for RUNX1 consensus sites using the Matlnspector promoter analysis tool. For reporter gene assay, $251 \mathrm{bp}$ of human RUNX1 intron 5 or $312 \mathrm{bp}$ of RUNX1 exon 1 were cloned by PCR ligation into the pGL3 promoter vector (Promega, Madison, WI, USA) upstream of SV40 promoter. The KRT1 ( $-846 /$ $+257)$ and LOR $(-1370 /+9)$ promoter segments were cloned into pGL4-basic vector (Promega). Deletion of the distal (D) or proximal (P) RUNX1 binding sites was performed by PCR/digestion from the full KRT1 promoter into pGL4-basic vector to construct the P-site KRT1 promoter $(-546 /+257)$ and the D-site KRT1 promoter $(-846 /+28)$ vectors, respectively. Transient transfections were carried out four times in duplicate on HEK293 cells using JetPei reagent (Polyplus). Luciferase assays were performed using Dual-Luciferase Reporter Assay System (Promega) according to the manufacturer instructions. The ratio of luciferase activity for intron5-pGL3 promoter and exon1-pGL3 promoter was calculated $24 \mathrm{~h}$ after transfection with empty pCMV, pCMV-p53, empty pcDNA3 or pcDNA3$\triangle N p 63^{40}$ relative to $\mathrm{pGL} 3$ promoter empty vector. For RUNX1 transfections, the luciferase activity ratio was calculated $24 \mathrm{~h}$ after transfection by $\mathrm{pCMV}-\mathrm{CBF} \beta$, pCMV-RUNX1 or both relative to pGL4-basic empty vector. Data were normalized to the transfection efficiency assessed by $\mathrm{PCMV}-\mathrm{RL}$ vector cotransfection.

Statistical analysis. All data are represented as a mean for at least three experiments. Statistical significance was calculated by a two-tailed Student's $t$-test for unpaired samples. Mean differences were considered to be significant when $P<0.05$ ( ${ }^{\star} P<0.05 ;{ }^{* \star} P<0.01 ;{ }^{* \star} P<0.001$; ns, not significant).

\section{Conflict of Interest}

The authors declare no conflict of interest.

Acknowledgements. We thank Dr. Dawhara and Dr. Clavel (Service d'Urologie, Clinique du Tonkin, Villeurbanne, France) for skin biopsies, Tamara Ducry for technical assistance and the ANIPATH platform for immunohistochemical processing. This work was supported by a grant from the French Society for Dermatological Research (SRD).

1. Koster MI, Roop DR. p63 and epithelial appendage development. Differentiation 2004; 72 : 364-370.

2. Mills $A A$, Zheng $B$, Wang $X J$, Vogel $H$, Roop DR, Bradley A. p63 is a p53 homologue required for limb and epidermal morphogenesis. Nature 1999; 398: 708-713.

3. Yang A, Schweitzer R, Sun D, Kaghad M, Walker N, Bronson RT et al. p63 is essential for regenerative proliferation in limb, craniofacial and epithelial development. Nature 1999; 398: $714-718$.

4. Truong AB, Kretz M, Ridky TW, Kimmel R, Khavari PA. p63 regulates proliferation and differentiation of developmentally mature keratinocytes. Genes Dev 2006; 20: 3185-3197.

5. Yang A, Kaghad M, Wang Y, Gillett E, Fleming MD, Dotsch V et al. p63, a p53 homolog at 3q27-29, encodes multiple products with transactivating, death-inducing, and dominant-negative activities. Mol Cell 1998; 2: 305-316.

6. King KE, Ponnamperuma RM, Gerdes MJ, Tokino T, Yamashita T, Baker CC et al. Unique domain functions of $\mathrm{p} 63$ isotypes that differentially regulate distinct aspects of epidermal homeostasis. Carcinogenesis 2006; 27: 53-63.

7. Kanitakis J, Chouvet B. Expression of p63 in cutaneous metastases. Am J Clin Pathol 2007; 128: 753-758.

8. Stransky N, Egloff AM, Tward AD, Kostic AD, Cibulskis K, Sivachenko A et al. The mutational landscape of head and neck squamous cell carcinoma. Science 2011; 333: 1157-1160.

9. Ortt K, Raveh E, Gat U, Sinha S. A chromatin immunoprecipitation screen in mouse keratinocytes reveals Runx1 as a direct transcriptional target of DeltaNp63. J Cell Biochem 2008; 104: 1204-1219. 
10. Pozzi S, Zambelli F, Merico D, Pavesi G, Robert A, Maltere P et al. Transcriptional network of p63 in human keratinocytes. PLOS One 2009; 4: e5008.

11. Vigano MA, Lamartine J, Testoni B, Merico D, Alotto D, Castagnoli $C$ et al. New p63 targets in keratinocytes identified by a genome-wide approach. EMBO J 2006; 25: 5105-5116.

12. Lutterbach $B$, Hiebert SW. Role of the transcription factor AML-1 in acute leukemia and hematopoietic differentiation. Gene 2000; 245: 223-235.

13. Blyth K, Cameron ER, Neil JC. The RUNX genes: gain or loss of function in cancer. Nat Rev Cancer 2005; 5: 376-387

14. Friedman AD. Cell cycle and developmental control of hematopoiesis by Runx1. J Cell Physiol 2009; 219: 520-524.

15. Wang X, Blagden C, Fan J, Nowak SJ, Taniuchi I, Littman DR et al. Runx1 prevents wasting, myofibrillar disorganization, and autophagy of skeletal muscle. Genes Dev 2005; 19: $1715-1722$.

16. Theriault FM, Nuthall HN, Dong Z, Lo R, Barnabe-Heider F, Miller FD et al. Role for Runx in the proliferation and neuronal differentiation of selected progenitor cells in the mammalian nervous system. J Neurosci 2005; 25: 2050-2061.

17. Hoi CS, Lee SE, Lu SY, McDermitt DJ, Osorio KM, Piskun CM et al. Runx1 directly promotes proliferation of hair follicle stem cells and epithelial tumor formation in mouse skin. Mol Cell Biol 2010; 30: 2518-2536.

18. Osorio KM, Lee SE, McDermitt DJ, Waghmare SK, Zhang YV, Woo HN et al. Runx1 modulates developmental, but not injury-driven, hair follicle stem cell activation. Development 2008; 135: 1059-1068.

19. Osorio KM, Lilja KC, Tumbar T. Runx1 modulates adult hair follicle stem cell emergence and maintenance from distinct embryonic skin compartments. J Cell Biol 2011; 193 235-250.

20. Soma T, Ishimatsu-Tsuji Y, Tajima M, Kishimoto J. Runx1 transcription factor is involved in the regulation of KAP5 gene expression in human hair follicles. J Dermatol Sci 2006; 41 221-224.

21. Inrie RA, Marques MR, Nguyen BT, Horner JS, Papazoglu C, Bronson RT et al. Perp is a p63-regulated gene essential for epithelial integrity. Cell 2005; 120: 843-856.

22. Lapi E, lovino A, Fontemaggi G, Soliera AR, lacovelli S, Sacchi A et al. S100A2 gene is a direct transcriptional target of p53 homologues during keratinocyte differentiation. Oncogene 2006; 25: 3628-3637.

23. Datto MB, Li Y, Panus JF, Howe DJ, Xiong Y, Wang XF. Transforming growth factor beta induces the cyclin-dependent kinase inhibitor p21 through a p53-independent mechanism. Proc Natl Acad Sci USA 1995; 92: 5545-5549.

24. Arman M, Aguilera-Montilla N, Mas V, Puig-Kroger A, Pignatelli M, Guigo R et al. The human CD6 gene is transcriptionally regulated by RUNX and Ets transcription factors in T cells. Mol Immunol 2009; 46: 2226-2235.

25. Toulza E, Mattiuzzo NR, Galliano MF, Jonca N, Dossat C, Jacob D et al. Large-scale identification of human genes implicated in epidermal barrier function. Genome Biol 2007; 8: R107.

26. Youssef KK, Van Keymeulen A, Lapouge G, Beck B, Michaux C, Achouri Y et al Identification of the cell lineage at the origin of basal cell carcinoma. Nat Cell Biol 2010; 12 299-305.
27. Lapouge G, Youssef KK, Vokaer B, Achouri Y, Michaux C, Sotiropoulou PA et al. Identifying the cellular origin of squamous skin tumors. Proc Natl Acad Sci USA 2011; 108: $7431-7436$.

28. Pfeifer GP, Besaratinia A. Mutational spectra of human cancer. Hum Genet 2009; 125: 493-506.

29. Park HR, Min SK, Cho HD, Kim KH, Shin HS, Park YE. Expression profiles of p63, p53, survivin, and hTERT in skin tumors. J Cutan Pathol 2004; 31: 544-549.

30. Kamiya M, Takeuchi Y, Katho M, Yokoo H, Sasaki A, Nakazato Y. Expression of p73 in normal skin and proliferative skin lesions. Pathol Int 2004; 54: 890-895.

31. De Laurenzi V, Rossi A, Terrinoni A, Barcaroli D, Levrero M, Costanzo A et al. p63 and p73 transactivate differentiation gene promoters in human keratinocytes. Biochem Biophys Res Commun 2000; 273: 342-346.

32. Cordani N, Pozzi S, Martynova E, Fanoni D, Borrelli S, Alotto D et al. Mutant p53 subverts p63 control over KLF4 expression in keratinocytes. Oncogene 2011; 30: 922-932.

33. Planaguma J, Gonzalez M, Doll A, Monge M, Gil-Moreno A, Baro T et al. The up-regulation profiles of p21WAF1/CIP1 and RUNX1/AML1 correlate with myometrial infiltration in endometrioid endometrial carcinoma. Hum Pathol 2006; 37: 1050-1057.

34. Huitfeldt HS, Heyden A, Clausen OP, Thrane EV, Roop D, Yuspa SH. Altered regulation of growth and expression of differentiation-associated keratins in benign mouse skin tumors. Carcinogenesis 1991; 12: 2063-2067.

35. Ogawa E, Okuyama R, Egawa T, Nagoshi H, Obinata M, Tagami H et al. p63/p51-induced onset of keratinocyte differentiation via the c-Jun $\mathrm{N}$-terminal kinase pathway is counteracted by keratinocyte growth factor. J Biol Chem 2008; 283: 34241-34249.

36. Tsai KY, Tsao H. The genetics of skin cancer. Am J Med Genet C Semin Med Genet 2004; 131C: 82-92.

37. de Gruijl FR, Rebel H. Early events in UV carcinogenesis-DNA damage, target cells and mutant p53 foci. Photochem Photobiol 2008; 84: 382-387.

38. Lamartine J, Franco N, Le Minter P, Soularue P, Alibert O, Leplat JJ et al. Activation of an energy providing response in human keratinocytes after gamma irradiation. J Cell Biochem 2005; 95: 620-631.

39. Bonin F, Molina M, Malet C, Ginestet C, Berthier-Vergnes O, Martin MT et al. GATA3 is a master regulator of the transcriptional response to low-dose ionizing radiation in human keratinocytes. BMC Genomics 2009; 10: 417.

40. Petitjean A, Ruptier C, Tribollet V, Hautefeuille A, Chardon F, Cavard C et al. Properties of the six isoforms of p63: p53-like regulation in response to genotoxic stress and cross talk with DeltaNp73. Carcinogenesis 2008; 29: 273-281.

Cell Death and Disease is an open-access journal published by Nature Publishing Group. This work is licensed under the Creative Commons Attribution-NonCommercial-No Derivative Works 3.0 Unported License. To view a copy of this license, visit http://creativecommons.org/licenses/by-nc-nd/3.0/

Supplementary Information accompanies the paper on Cell Death and Disease website (http://www.nature.com/cddis) 